\title{
Local Centrally Essential Subalgebras of Triangular Algebras
}

\author{
O.V. Lyubimtsev, A.A. Tuganbaev
}

Key words: centrally essential algebra, algebra of upper triangular matrices, nilpotence index.

\begin{abstract}
We study local centrally essential subalgebras in the algebra of all upper triangular matrices over a field of characteristic $\neq 2$. It is proved that the algebras of upper triangular $3 \times 3$ or $4 \times 4$ matrices have only commutative local centrally essential subalgebras. Every algebra of upper triangular matrices of order exceeding 6 contains a non-commutative local centrally essential subalgebra.

The work of O.V. Lyubimtsev is done under the state assignment No 07292020-0055. A.A. Tuganbaev is supported by Russian Scientific Foundation, project 16-11-10013P.
\end{abstract}

\section{MSC2010 database 16R99; 20K30}

\section{Introduction}

We only consider associative rings which are not necessarily unital.

\subsection{Centrally essential rings.}

A ring $R$ is said to be centrally essential if either it is commutative or for any non-central element $a \in R$, there exist two non-zero central elements $x, y$ with $a x=y$. A ring $R$ with non-zero identity element is centrally essential if and only if the following condition $(*)$ holds:

for any non-zero element $a \in R$, there exist two non-zero central elements $x, y \in R$ with $a x=y$. Any non-zero ring with zero multiplication is centrally essential but does not satisfy $(*)[1$

Centrally essential rings with non-zero identity element are studied in papers [6], [7], 8], [9], [10], [11], [12]. Every centrally essential semiprime ring with $1 \neq 0$ is commutative; see [6, Proposition 3.3]. In [6], examples of noncommutative group algebras over fields are given. For example, if $Q_{8}$ is the quaternion group of order 8 , then its group algebra over the field of order 2 is a finite local non-commutative centrally essential ring of order 256 . In

\footnotetext{
${ }^{1}$ Not necessarily unital rings with $(*)$ are considered in [13].
} 
addition, in [7] it is proved that the external algebra of a three-dimensional linear space over the field of order 3 is a finite non-commutative centrally essential ring, as well. In [9], there is an example of a centrally essential ring $R$ with $1 \neq 0$ such that the factor ring of $R$ with respect to the prime radical is not a PI ring. Abelian groups with centrally essential endomorphism rings are considered in [5].

The main result of this paper is Theorem 1.2.

1.2. Theorem. For any field $\mathbb{F}$ of characteristic $\neq 2$ and an arbitrary positive integer $n \geq 7$, there exists a local non-commutative centrally essential subalgebra of the algebra $T_{n}(\mathbb{F})$ of upper triangular $n \times n$ matrices.

1.3. Remark. For $n \geq 2$ and a field $F$, the complete matrix algebra $M_{n}(\mathbb{F})$ over $F$ and the algebra $T_{n}(\mathbb{F})$ of upper triangular matrices over $F$ are not centrally essential, since all idempotents of any centrally essential ring with $1 \neq 0$ are central by [6, Lemma 2.3].

An algebra $\mathcal{A}$ is said to be centrally essential if $\mathcal{A}$ is a centrally essential ring. In this paper, we consider local centrally essential subalgebras of the algebra $T_{n}(\mathbb{F})$ of all upper triangular matrices, where $\mathbb{F}$ is a field of characteristic $\neq 2$. In particular, such subalgebras are of interest, since, for $\mathbb{F}=\mathbb{Q}$, they are quasi-endomorphism algebras of strongly indecomposable torsionfree Abelian groups of finite rank $n$. Quasi-endomorphism algebras of all such groups are local matrix subalgebras in algebra $M_{n}(\mathbb{Q})$ of all matrices of order $n$ over the field $\mathbb{Q}$; e.g., see [4, Chapter I, §5]. We remark that the algebra $\mathbb{Q} E$ is the quasi-endomorphism algebra of a strongly indecomposable torsion-free Abelian group of prime rank $p$ if and only if $\mathbb{Q} E$ is isomorphic to a local subalgebra of $T_{p}(\mathbb{Q})$. Indeed, $\mathbb{Q} E / J(\mathbb{Q} E) \cong \mathbb{Q}$ in this case; see $[2$, Theorem 4.4.12], where $J(\mathbb{Q} E)$ is the Jacobson radical which is nilpotent, since $\mathbb{Q} E$ is Artinian. It follows from the Weddenburn-Malcev theorem 2 that $\mathbb{Q} E \cong \mathbb{Q} E_{p} \bigoplus J(\mathbb{Q} E)$, where $E_{p}$ is the identity matrix. It is known that every nilpotent subalgebra of a full matrix algebra $M_{n}(\mathbb{F})$ over an arbitrary field $\mathbb{F}$ is transformed by conjugation to a nil-triangular subalgebra; see [15, Chapter 2, Theorem 6]. Since diagonal matrices of a local matrix algebra have equal elements on the main diagonal, they are transformed to itself under conjugation. Consequently, the quasi-endomorphism algebras of such Abelian groups can be realized as matrix subalgebras if and only if these subalgebras are conjugated to some local subalgebra of $T_{p}(\mathbb{Q})$. The necessary information on Abelian groups can be found in [3] and [4].

Let $\mathbb{F}$ be a field and $\mathcal{A}$ a finite-dimensional algebra over $\mathbb{F}$. An element $a$ of

\footnotetext{
${ }^{2}$ See, for example, [1, Theorem 6.2.1].
} 
the algebra $\mathcal{A}$ is said to be nilpotent if $a^{n}=0$ for some $n \in \mathbb{N}$. The minimal value of $n$ with this property is called the nilpotence index of the element a. An algebra $\mathcal{A}$ is called a nil-algebra if every element is nilpotent. For a nil-algebra $\mathcal{A}$, the maximal nilpotence index $\nu(\mathcal{A})$ of its elements is called the nil-index. A positive integer $k$ such that $\mathcal{A}^{k}=(0)$ and $\mathcal{A}^{k-1} \neq(0)$ is called the nilpotence index of the algebra $\mathcal{A}$. If such an integer $k$ exists, then algebra is said to be of nilpotent index $k$.

For a field $\mathbb{F}$ and an associative algebra $\mathcal{A}$ over $\mathbb{F}$, we denote by $Z(\mathcal{A})$ and $J(\mathcal{A})$ the center and the Jacobson radical of the algebra $\mathcal{A}$, respectively. An algebra $\mathcal{A}$ with 1 is said to be local if the factor algebra $\mathcal{A} / J(\mathcal{A})$ is a division algebra. Further, $\mathcal{A}$ denotes a local subalgebra in the algebra $T_{n}(\mathbb{F})$ and $N_{n}(\mathbb{F})$ denotes the subalgebra of nilpotent matrices in $\mathcal{A}$ (i.e., the algebra of strictly upper triangular matrices). We note that any matrix $A \in \mathcal{A}$ is of the form

$$
A=\left(\begin{array}{cccc}
\lambda & a_{12} & \ldots & a_{1 n} \\
0 & \lambda & \ldots & a_{2 n} \\
\vdots & \vdots & \ddots & \vdots \\
0 & 0 & \ldots & \lambda
\end{array}\right)
$$

We denote by $E_{i j}$ the matrix unit, i.e., the matrix with 1 on the position $(i, j)$ and zeros on the remaining positions; $E_{k}$ denotes the identity $k \times k$ matrix. We denote by $\langle S\rangle$ the linear hull of a subset $S$ of some linear space.

\section{Some General Results}

We recall that a ring $R$ is said to be semiprime if $R$ does not contain two non-zero ideals with zero multiplication. A ring is said to be reduced if it does not contain zero-square elements. The center of a semiprime ring is a reduced ring.

2.1. Proposition. If $R$ is a centrally essential ring and its center is a semiprime ring, then the ring $R$ is commutative.

Proof. This assertion follows from [13, Theorem 1.3(a)] in the case, where $R$ satisfies condition $1.1(*)$.

We consider the general case. Suppose that the $\operatorname{ring} R$ is not commutative, i.e., there exist $x, y \in R$ such that $x y-y x \neq 0$. Since $R$ is a centrally essential ring and $Z(R)$ is a reduced ring, there exist $c, d \in Z(R)$ such that $d=(x y-y x) c \in Z(R) \backslash 0$. We note that $x d \neq 0$; otherwise $d^{2}=(x y-y x) c d=$ 
$((x d) y-y(x d)) c=0$, which is impossible. If $x d \notin Z(R)$, then we can repeat the proof of [13, Theorem 2.1(c)]. Namely, there exists an element $z \in Z(R)$ such that $x d z \in Z(R) \backslash 0$. We consider the set $I=\{i \in Z(R) \mid i x \in Z(R)\}$. It is clear that $d z \in I$. Now assume that $d I=0$. Then $d(d z)=0,(d z)^{2}=0$ and $d z=0$; this is a contradiction. Therefore, $d i \neq 0$ for some $i \in I$. However,

$$
d i=(x y-y x) c i=((i x) y-y(i x)) c=0,
$$

and we obtain a contradiction, as well.

We assume that $x d \in Z(R)$. Then $x d y \neq 0$, since otherwise $d^{2}=0$. In addition, $(x y) d=(y x) d$. Therefore, $(x y-y x) d=0$. However, then we have $d^{2}=(x y-y x) c d=0$; this is a contradiction. Thus, the ring $R$ is commutative.

The following assertion expands Lemma 2.3 of [6] to the case of rings which do not necessarily have $1 \neq 0$; in addition, 1 is replaced by an arbitrary idempotent $e$.

2.2. Proposition. In any centrally essential ring, the following condition holds:

$$
\begin{gathered}
\forall n \in \mathbb{N}, x_{1}, \ldots, x_{n}, y_{1}, \ldots, y_{n}, r, e=e^{2} \in R, \\
\left\{\begin{array}{l}
x_{1} y_{1}+\ldots+x_{n} y_{n}=e \\
x_{1} r e y_{1}+\ldots+x_{n} r e y_{n}=0
\end{array} \Rightarrow r e=0 .\right.
\end{gathered}
$$

In particular, all idempotents of a centrally essential ring are central.

Proof. We assume that $R$ is a centrally essential ring which satisfies condition (1), but $r e \neq 0$. If $r e \in Z(R)$, then

$$
r e=r e^{2}=r e\left(x_{1} y_{1}+\ldots+x_{n} y_{n}\right)=x_{1} r e y_{1}+\ldots+x_{n} r e y_{n}=0 ;
$$

this is a contradiction.

Let $r e \notin Z(R)$. Then there exist two elements $c, d \in Z(R)$ such that cre = $d \neq 0$. We note that $d=$ cre $=($ cre $) e=d e$. Therefore,

$$
d=d e=d\left(x_{1} y_{1}+\ldots+x_{n} y_{n}\right)=c\left(x_{1} r e y_{1}+\ldots+x_{n} r e y_{n}\right)=0 .
$$

Now let $e=e^{2} \notin Z(R)$. We have the relations $e \cdot e=e$ and $e(r e) e=0$, where $r=x-e x$ for any $x \in R$. Consequently, $r e=0$ and $x e=e x e$. We note that condition (1) remains true if we replace re by $e r$. In this case, ex $=e x e$. Therefore, all idempotents of the ring $R$ are central.

2.3. Proposition. Let $\mathcal{A}$ be a local subalgebra of $T_{n}(\mathbb{F})$ with Jacobson radical $J(\mathcal{A})$. The algebra $\mathcal{A}$ is centrally essential if and only if $J(\mathcal{A})$ is a centrally essential algebra. 
Proof. Let us have a matrix $A \in J(\mathcal{A})$ with $A \notin Z(J(\mathcal{A}))$. Since $\mathcal{A}$ is a centrally essential algebra, there exists a matrix $B \in Z(\mathcal{A})$ such that $0 \neq A B=C \in Z(\mathcal{A})$. Since $J(\mathcal{A})$ is an ideal, $C \in Z(J(\mathcal{A}))$. If $B \notin J(\mathcal{A})$, then $A=C B^{-1} \in Z(J(\mathcal{A}))$; this contradicts to the choice of the matrix $A$.

Conversely, we have a decomposition $\mathcal{A}=\mathbb{F} E_{n} \bigoplus J(\mathcal{A})$. Since $\mathbb{F} E_{n} \subset Z(\mathcal{A})$,

$$
Z(J(\mathcal{A})) \subset Z(\mathcal{A}) .
$$

If $0 \neq A \in \mathcal{A}$ and $A \in Z(\mathcal{A})$, then $0 \neq A E_{n} \in Z(\mathcal{A})$. Let $A \notin Z(\mathcal{A})$ and $A \in J(\mathcal{A})$. Then there exists a matrix $B \in Z(J(\mathcal{A}))$ such that $0 \neq A B=$ $C \in Z(J(\mathcal{A}))$. It follows from relation $(2)$ that $B \in Z(\mathcal{A})$ and $C \in Z(\mathcal{A})$.

Let $A \notin J(\mathcal{A})$. Then $A=A^{\prime}+A^{\prime \prime}$, where $0 \neq A^{\prime} \in \mathbb{F} E_{n}, A^{\prime \prime} \in J(\mathcal{A})$. If $A^{\prime \prime}=0$, then $A \in Z(\mathcal{A})$. Otherwise, $0 \neq A^{\prime \prime} B \in Z(J(\mathcal{A}))$ for some matrix $B \in Z(J(\mathcal{A}))$. Then

$$
A B=A^{\prime} B+A^{\prime \prime} B=B A^{\prime}+B A^{\prime \prime}=B A .
$$

Since $A^{\prime} B, A^{\prime \prime} B \in Z(J(\mathcal{A}))$, we have $A B \in Z(J(\mathcal{A})) \subset Z(\mathcal{A})$. We also note that $A B \neq 0$, since the matrix $A$ is invertible.

By considering Remark 1.3, we obtain the following corollary.

2.4. Corollary. The algebra $N_{n}(\mathbb{F})$ for $n \geq 3$ is not a centrally essential algebra.

It follows from Proposition 2.3 that the problem of constructing local centrally essential subalgebras of the algebra $T_{n}(\mathbb{F})$ is equivalent to the problem of constructing centrally essential subalgebras of the algebra $N_{n}(\mathbb{F})$.

Let $\mathcal{A}$ be a subalgebra of the algebra $N_{n}(\mathbb{F})$ of nilpotence index $n$. We assume that $\nu(\mathcal{A})=n$. Then there exists a matrix $A \in \mathcal{A}$ such that $A^{n-1} \neq 0$. We transform $A$ to the Jordan form,

$$
A=E_{12}+E_{23}+\ldots+E_{(n-1) n},
$$

and pass to the corresponding conjugated subalgebra $\mathcal{A}_{c}$. We denote by $C(A)$ the centralizer of the matrix $A$ in $\mathcal{A}_{c}$. Since the minimal polynomial of the matrix $A$ is equal to its characteristic polynomial, $C(A)=\mathbb{F}[A]$, where $\mathbb{F}[A]$ is the ring of all matrices which can be represented in the form $f(A)$, $f(x) \in \mathbb{F}[x]$; see [15, Chapter 1 , Theorem 5]. For $B \in C(A)$, we have

$$
B=f(A)=\alpha_{0} E_{n}+\alpha_{1} A+\ldots+\alpha_{n-1} A^{n-1} .
$$

In addition, $\alpha_{0}=0$, since the matrix $B$ is nilpotent. 
2.5. Lemma. If $Z\left(\mathcal{A}_{c}\right)=C(A)$, then the algebra $\mathcal{A}_{c}$ is commutative.

Proof. Indeed, if $A^{\prime} \notin C(A)$, then $A A^{\prime} \neq A^{\prime} A$. However, $A \in C(A)=$ $Z\left(\mathcal{A}_{c}\right)$. This is a contradiction.

2.6. Lemma. Let $\mathcal{A}_{c}$ be a centrally essential algebra and $Z\left(\mathcal{A}_{c}\right)=<A^{n-1}>$. Then the algebra $\mathcal{A}_{c}$ is commutative.

Proof. Indeed, if $\mathcal{A}_{c}$ is not commutative, then for the matrix $A^{\prime} \notin Z\left(\mathcal{A}_{c}\right)$ we have $B A^{\prime}=0$ for any matrix $B \in Z\left(\mathcal{A}_{c}\right)$.

\section{Nilpotent Centrally Essential Subalgebras of Algebras $N_{3}(\mathbb{F})$ and $N_{4}(\mathbb{F})$}

In what follows, we assume that the ground field $\mathbb{F}$ is of characteristic $\neq 2$.

3.1. Proposition. Any centrally essential subalgebra of the algebra $N_{3}(\mathbb{F})$ is commutative.

Proof. Every matrix $A \in N_{3}(\mathbb{F})$ is of the form

$$
A=\left(\begin{array}{ccc}
0 & a & b \\
0 & 0 & c \\
0 & 0 & 0
\end{array}\right) .
$$

Let $\mathcal{A}$ be a non-commutative centrally essential subalgebra of the algebra $N_{3}(\mathbb{F})$ of nilpotence index 3. Then $\nu(\mathcal{A})=3$. Let a matrix $A \in \mathcal{A}$ be of nilpotence index 3. We transform $A$ to the Jordan form: $A=E_{12}+E_{23}$. Now, if $B \in C(A)$, then $B=\alpha_{1} A+\alpha_{2} A^{2}$. We note that $Z\left(\mathcal{A}_{c}\right) \subseteq C(A)$; in addition, $\nu\left(Z\left(\mathcal{A}_{c}\right)\right)=3$ by Lemma 2.6. However, then $Z\left(\mathcal{A}_{c}\right)=C(A)$ and the algebra $\mathcal{A}_{c}$ is commutative by Lemma 2.5. This is a contradiction.

In particular, it follows from Proposition 3.1 that all centrally essential endomorphism rings of strongly indecomposable Abelian torsion-free groups of rank 3 are commutative; cf. [5. Example 3.4].

3.2. Proposition. Any centrally essential subalgebra $\mathcal{A}$ of the algebra $N_{4}(\mathbb{F})$ is commutative.

Proof. If the algebra $\mathcal{A}$ is of nilpotence index 2, then it is commutative. Let the nilpotence index of $\mathcal{A}$ be equal to 4 . There exists $A \in \mathcal{A}$ such that $A^{3} \neq 0$. Indeed, the algebra $\mathcal{A}$ contains three matrices $S=\left(s_{i j}\right), T=\left(t_{i j}\right)$, $P=\left(p_{i j}\right)$ with $s_{12} \neq 0, t_{23} \neq 0, p_{34} \neq 0$. Otherwise, the nilpotence index $\mathcal{A}$ is lower than 4 . As the required matrix, we can take a matrix $A=\left(a_{i j}\right)$ such 
that $a_{i(i+1)} \neq 0, i=1,2,3$. We transform $A$ to the Jordan form,

$$
A=E_{12}+E_{23}+E_{34}
$$

and pass to the corresponding conjugated subalgebra $\mathcal{A}_{c}$. For a matrix $B \in$ $C(A)$, we have

$$
B=\alpha_{1} A+\alpha_{2} A^{2}+\alpha_{3} A^{3},
$$

where $\alpha_{1}, \alpha_{2}, \alpha_{3} \in \mathbb{F}$. It follows from Lemma 2.5 and Lemma 2.6, then $Z\left(\mathcal{A}_{c}\right) \neq C(A)$ and $Z\left(\mathcal{A}_{c}\right) \neq<A^{3}>$ provided the algebra $\mathcal{A}_{c}$ is not commutative. Then any matrix $C \in Z\left(\mathcal{A}_{c}\right)$ is of the form

$$
C=\left(\begin{array}{cccc}
0 & 0 & c_{13} & c_{14} \\
0 & 0 & 0 & c_{13} \\
0 & 0 & 0 & 0 \\
0 & 0 & 0 & 0
\end{array}\right)
$$

Since $\mathcal{A}_{c}$ is a centrally essential algebra, we have that for the non-zero matrix $D \notin Z\left(\mathcal{A}_{c}\right)$, there exists a matrix $C \in Z\left(\mathcal{A}_{c}\right)$ such that $0 \neq D C \in Z\left(\mathcal{A}_{c}\right)$. Since the matrix $D$ is nilpotent, $\operatorname{tr} D=0$. In addition, $\mathcal{A}_{c}$ is local; therefore all elements on the main diagonal of the matrix $D$ are equal to zero. In this case, it is directly calculated that

$$
D=\left(\begin{array}{cccc}
0 & d_{12} & d_{13} & d_{14} \\
0 & 0 & d_{23} & d_{24} \\
0 & 0 & 0 & d_{12} \\
0 & 0 & 0 & 0
\end{array}\right)
$$

If $d_{12}=0$ and $D \notin Z\left(\mathcal{A}_{c}\right)$, then $D C=0$ for any matrix $C \in Z\left(\mathcal{A}_{c}\right)$; this is a contradiction. Let $d_{12} \neq 0$ and $D F \neq F D$ for some matrix $F=\left(f_{i j}\right) \in \mathcal{A}_{c}$. We find an element $\lambda \in \mathbb{F}$ such that $f_{12}=\lambda d_{12}$. We set $G=\lambda D-F$, $G=\left(g_{i j}\right)$. Then

$$
\begin{aligned}
& F G=F(\lambda D-F)=\lambda F D-F^{2}, \\
& G F=(\lambda D-F) F=\lambda D F-F^{2} .
\end{aligned}
$$

Therefore, $G \notin Z\left(\mathcal{A}_{c}\right)$ and $g_{12}=0$. It follows from the obtained contradiction that the algebra $\mathcal{A}_{c}$ is commutative.

Let the nilpotence index of the algebra $\mathcal{A}$ be equal to 3 . Then $\nu(\mathcal{A})=3$, i.e., $\mathcal{A}$ contains a matrix $A$ such that $A^{2} \neq 0$. Indeed, let us assume the contrary, $A^{2}=0$ for all $A \in \mathcal{A}$. If $A \notin Z(\mathcal{A})$, then $0 \neq A B \in Z(\mathcal{A})$ for some matrix $B \in Z(\mathcal{A})$. Then

$$
(A+B)^{2}=A^{2}+2 A B+B^{2}=2 A B=0 .
$$


Hence $A B=0$. This is a contradiction.

We transform the matrix $A$ to the Jordan form,

$$
A=E_{12}+E_{23} \text {. }
$$

In the corresponding conjugated subalgebra $\mathcal{A}_{c}$, the centralizer $C(A)$ consists of matrices $B$ of the form

$$
B=\left(\begin{array}{cccc}
0 & b_{12} & b_{13} & b_{14} \\
0 & 0 & b_{12} & 0 \\
0 & 0 & 0 & 0 \\
0 & 0 & b_{43} & 0
\end{array}\right)
$$

see [15, Chapter $3, \S 1]$. In addition, if $C \in Z(C(A))$, then we have

$$
C=\left(\begin{array}{cccc}
0 & c_{12} & c_{13} & 0 \\
0 & 0 & c_{12} & 0 \\
0 & 0 & 0 & 0 \\
0 & 0 & 0 & 0
\end{array}\right)
$$

Let $Z\left(\mathcal{A}_{c}\right)$ be of nilpotence index 3 . Then we can take a matrix from $Z\left(\mathcal{A}_{c}\right)$ as the matrix $A$; see [15, Chapter 1, Proposition 5, Corollary]. In this case, all matrices from $\mathcal{A}_{c}$ are contained in $C(A)$. Then $\mathcal{A}_{c}$ consists of the matrices of the form (3) and the matrices from $Z\left(\mathcal{A}_{c}\right)$ is form (4). If $B=\left(b_{i j}\right) \notin Z\left(\mathcal{A}_{c}\right)$ and $b_{12}=0$, then $B C=0$ for all $C \in Z\left(\mathcal{A}_{c}\right)$. Then $\mathcal{A}_{c}$ is not a centrally essential algebra. Let $b_{12} \neq 0$ and $B D \neq D B$ for some matrix $D=\left(d_{i j}\right) \in \mathcal{A}_{c}$. Let $d_{12}=\lambda b_{12}$ and $F=\lambda B-D, F=\left(f_{i j}\right)$. Then $f_{12}=0$ and $F \notin Z\left(\mathcal{A}_{c}\right)$. This is a contradiction.

Let $Z\left(\mathcal{A}_{c}\right)$ be of nilpotence index 2. Then for $C \in Z\left(\mathcal{A}_{c}\right)$, we obtain

$$
C=\left(\begin{array}{cccc}
0 & 0 & c_{13} & 0 \\
0 & 0 & 0 & 0 \\
0 & 0 & 0 & 0 \\
0 & 0 & 0 & 0
\end{array}\right) .
$$

It follows from relation $A C=C A$ for $A \in \mathcal{A}_{c}$ that

$$
A=\left(\begin{array}{cccc}
0 & a_{12} & a_{13} & a_{14} \\
0 & 0 & a_{23} & a_{24} \\
0 & 0 & 0 & 0 \\
0 & a_{42} & a_{43} & 0
\end{array}\right) .
$$

However, then $A C=0$ for any matrix $C \in Z\left(\mathcal{A}_{c}\right)$. Consequently, if $\mathcal{A}_{c}$ is a centrally essential algebra, then $\mathcal{A}_{c}$ is commutative. 


\section{The Proof of Theorem 1.2}

In [7, Proposition 2.5], it is proved that the external algebra $\Lambda(V)$ over a field $\mathbb{F}$ of characteristic $\neq 2$ is a centrally essential algebra if and only if the dimension of the space $V$ is odd. By considering the regular matrix representation of the algebra $\Lambda(V)$, we obtain that for an odd positive integer $n>1$, there exists a non-commutative centrally essential subalgebra of the algebra $N_{2^{n}}(\mathbb{F})$; also see [5, Example 3.5]. Therefore, the minimal order of matrices of a non-commutative the external centrally essential algebra is equal to 8 . In the next example, we construct a non-commutative centrally essential algebra of $7 \times 7$ matrices.

4.1. Example. We consider a subalgebra $\mathcal{A}$ of $N_{7}(\mathbb{F})$ consisting of matrices $A$ of the form

$$
A=\left(\begin{array}{lllllll}
0 & a & b & c & d & e & f \\
0 & 0 & 0 & b & 0 & 0 & d \\
0 & 0 & 0 & 0 & 0 & 0 & e \\
0 & 0 & 0 & 0 & 0 & 0 & 0 \\
0 & 0 & 0 & 0 & 0 & 0 & a \\
0 & 0 & 0 & 0 & 0 & 0 & b \\
0 & 0 & 0 & 0 & 0 & 0 & 0
\end{array}\right)
$$

Let $A^{\prime} \in \mathcal{A}, a^{\prime}=a+1$ and the remaining components of the matrix $A^{\prime}$ coincide with the corresponding components of the matrix $A$. Then $A A^{\prime} \neq$ $A^{\prime} A$ if $a \neq 0$ and $b \neq 0$. Therefore, the algebra $\mathcal{A}$ is not commutative. It is easy to see that $Z(\mathcal{A})$ contain matrices $B$ of the form

$$
B=\left(\begin{array}{ccccccc}
0 & 0 & 0 & c & d & e & f \\
0 & 0 & 0 & 0 & 0 & 0 & d \\
0 & 0 & 0 & 0 & 0 & 0 & e \\
0 & 0 & 0 & 0 & 0 & 0 & 0 \\
\ldots & \ldots & \ldots & \ldots & \ldots & \ldots & \ldots \\
0 & 0 & 0 & 0 & 0 & 0 & 0
\end{array}\right)
$$

If $0 \neq A \notin Z(\mathcal{A})$, then $0 \neq A B \in Z(\mathcal{A})$ for some matrix $B \in Z(\mathcal{A})$. Consequently, $\mathcal{A}$ is a centrally essential algebra.

\subsection{The completion of the proof of Theorem 1.2 .}


In $N_{n}(\mathbb{F})$, we consider the subalgebra $\mathcal{A}$ matrices $A$ of the form

$$
A=\left(\begin{array}{ccccccccc}
0 & a_{12} & a_{13} & a_{14} & a_{15} & \ldots & a_{1 n-2} & a_{1 n-1} & a_{1 n} \\
0 & 0 & 0 & a_{13} & 0 & \ldots & 0 & 0 & a_{1 n-2} \\
0 & 0 & 0 & 0 & 0 & \ldots & 0 & 0 & a_{1 n-1} \\
0 & 0 & 0 & 0 & 0 & \ldots & 0 & 0 & 0 \\
\ldots & \ldots & \ldots & \ldots & \ldots & \ldots & \ldots & \ldots & \ldots \\
0 & 0 & 0 & 0 & 0 & \ldots & 0 & 0 & 0 \\
0 & 0 & 0 & 0 & 0 & \ldots & 0 & 0 & a_{12} \\
0 & 0 & 0 & 0 & 0 & \ldots & 0 & 0 & a_{13} \\
0 & 0 & 0 & 0 & 0 & \ldots & 0 & 0 & 0
\end{array}\right) .
$$

We remark that the algebra $\mathcal{A}$ is not commutative; also see Example 4.1. If $B \in Z(\mathcal{A})$, then

$$
B=\left(\begin{array}{ccccccccc}
0 & 0 & 0 & b_{14} & b_{15} & \ldots & b_{1 n-2} & b_{1 n-1} & b_{1 n} \\
0 & 0 & 0 & 0 & 0 & \ldots & 0 & 0 & b_{1 n-2} \\
0 & 0 & 0 & 0 & 0 & \ldots & 0 & 0 & b_{1 n-1} \\
0 & 0 & 0 & 0 & 0 & \ldots & 0 & 0 & 0 \\
\ldots & \ldots & \ldots & \ldots \ldots & \ldots & \ldots & \ldots & \ldots & \ldots \\
0 & 0 & 0 & 0 & 0 & \ldots & 0 & 0 & 0
\end{array}\right) .
$$

For $A=\left(a_{i j}\right) \notin Z(\mathcal{A})$, we have $a_{12} \neq 0, a_{13} \neq 0$. Let $B=\left(b_{i j}\right) \in Z(\mathcal{A})$ and $b_{1 n-2}=a_{12}, b_{1 n-1}=a_{13}$. Then $0 \neq A B \in Z(\mathcal{A})$. Indeed, let $A B=C=\left(c_{i j}\right)$, $B A=D=\left(d_{i j}\right)$. Then $c_{i j}=d_{i j}=0$ for all $i \neq 1, j \neq n$. In addition, $c_{1 n}=d_{1 n}=a_{12}^{2}+a_{13}^{2}$. Therefore, $\mathcal{A}$ is a centrally essential algebra.

It is known (e.g., see [14]) that every rational algebra of dimension $n$ can be realized as the quasi-endomorphism ring of torsion-free Abelian group of rank $n$. By considering [5, Proposition 3.1], we obtain the following corollary.

4.3. Corollary. For every positive integer $n>6$, there exists an Abelian torsion-free group $A(n)$ of rank $n$ such that its endomorphism ring is a noncommutative centrally essential ring.

\section{$5 \quad$ Remarks and Open Questions}

5.1. It follows from Proposition 2.3 that for a centrally essential local ring $R$, the ring $J(R)$ is centrally essential. The converse is not always true. Indeed, let $R$ be the local ring of upper triangular matrices $2 \times 2$ over a division ring $D$ which is not a field. Then $J(R)$ is a commutative ring with zero 
multiplication. However, the ring $R$ is not centrally essential, since the ring $R / J(R)$ is not commutative; see [7, Proposition 3.3].

5.2. Open question. Is it true that for any centrally essential ring $R$, the Jacobson radical $J(R)$ is a centrally essential ring?

5.3. Open question. In Theorem 1.2, it is proved that for any positive integer $n>6$, there exists a local non-commutative centrally essential subalgebra of the algebra $T_{n}(F)$ of nilpotence index 3 . Is it true that there exists a positive integer $n$ such that $T_{n}(F)$ contains a local non-commutative centrally essential subalgebra of any nilpotence index $2<k \leq n$ ?

5.4. Open question. Is it true that there exist local non-commutative centrally essential subalgebras of the algebra $T_{n}(F)$ for $n=5$ and $n=6$ ?

5.5. Open question. Is it true that for every positive integer $k>2$, there exists a positive integer $n=n(k)$ such that the algebra $T_{n}(F)$ contains a local non-commutative centrally essential subalgebra of nilpotence index $k$ ?

\section{References}

[1] Drozd Y. A., Kirichenko V. V. Finite Dimensional Algebras // SpringerVerlag Berlin Heidelberg, 1994.

[2] Faticoni T. Direct Sum Decompositions of Torsion-Free Finite Rank Groups // Taylor\&Francis Group, 2007.

[3] Fuchs L. Abelian groups // Springer Monographs in Mathematics, 2015.

[4] Krylov P.A., Mikhalev A.V., Tuganbaev A.A. Endomorphism rings of Abelian groups // Dordrecht/Boston/London, Springer Netherlands (Kluwer), 2003.

[5] Lyubimtsev O.V., Tuganbaev A.A. Centrally Essential Endomorphism Rings of Abelian Groups // Comm. Algebra. - DOI: 10.1080/00927872.2019.1677698. Also see Archive: http://arxiv.org/abs/1910.01222

[6] Markov V.T., Tuganbaev A.A. Centrally essential group algebras// J. Algebra. - 2018. - Vol. 512, no. 15. - P. 109-118.

[7] Markov V.T., Tuganbaev A.A. Centrally essential rings// Discrete Math. Appl. - 2019. - Vol. 29, no. 3. - P. 189-194. 
[8] Markov V.T., Tuganbaev A.A. Rings essential over their centers// Comm. Algebra. - 2019. - Vol. 47, no. 4. - P. 1642-1649.

[9] Markov V.T., Tuganbaev A.A. Rings with Polynomial Identity and Centrally Essential Rings// Beiträge zur Algebra und Geometrie / Contributions to Algebra and Geometry. - 2019. - DOI: https://doi.org/10.1007/s13366-019-00447-w

[10] Markov V.T., Tuganbaev A.A. Uniserial Artinian Centrally Essential Rings// Beitr. Algebra Geometrie / Contributions to Algebra and Geometry. - 2020. - Vol. 61, no. 1. - P. 23-33.

[11] Markov V.T., Tuganbaev A.A. Uniserial Noetherian Centrally Essential Rings// Comm. Algebra. - 2020. - Vol. 48, no. 1. - P. 149-153.

[12] Markov V.T., Tuganbaev A.A. Constructions of Centrally Essential Rings// Comm. Algebra. - 2019. https://doi.org/10.1080/00927872.2019.1635611.

[13] Markov V.T., Tuganbaev A.A. Centrally essential rings which are not necessarily unital or associative// Discrete Math. Appl. - 2019. - Vol. 29. - P. 215-218.

[14] Pierce R.S., Vinsonhaler C. Realizing central division algebras// Pacific J. Math. - 1983. - Vol. 109, no. 1. - P. 165-177.

[15] Suprunenko D.A., R.I. Tyschkevich R.I. Commutative Matrices// Academic Press, New York, 1968. 